\title{
Masticator Space Abscess: A Case Report
}

\author{
Dr G.V Thakur*,Dr V.T Kandakure**, Dr A.Thote\#, Dr Ayesha .K\# \\ *Associate Professor and Head, Department of Otorhinolaryngology, GMC, Latur,Maharashtra state, India \\ **Assistant Professor, Department of Otorhinolaryngology, GMC, Latur, Maharashtra state, India \\ \#Junior resident,department of Otorhinolaryngology. GMC Latur, Maharashtra state,India
}

\begin{abstract}
Different neoplasms and infections are known to involve the masticator space, but pathological diagnosis and treatment of these lesions are not always simple due to anatomical complexity. We treated a 65year-old woman with an abscess in the masticator space suffering from trismus and swelling over left cheek region and left-TMJ pain, which was initially diagnosed as a superficial cheek abscess. Masticator space abscesses have been reported more frequently in recent years. They are usually secondary to extractions of the first and second mandibular molar teeth Therefore, Successful aspiration of pus was followed by an instantaneous improvement in the ability to open the mouth for a period of at least 24 hours. The infection had spread upward along the mastication muscles, resulting in an abscess in both the upper masseter muscle and the lower temporalis muscle. Based on a review of the literature, most abscesses in the masticator space originate from the mandibular molar, while the most impressive physical finding varied between the submandibular region and temporal fossa, as did its acute or chronic clinical course. We emphasize diagnostic significance when assessing findings for each masticator muscle and mandibular bone depicted using computed tomography.
\end{abstract}

Key Words: abscess, CT scan, masticator abscess, trismus

\section{Introduction:}

Masticator space is a distinct deep facial space bounded by superficial layer of deep cervical fascia containing 4 muscles of mastication and ramus and posterior body of mandible.It lies anterolateral to the parapharyngeal space. The most common source of infection is dental in origin, most commonly the third molar. Due to close proximity of the muscules of mastication, patients with infections often have severe trismus as well as tenderness along the mandible. Jaw swelling and trismus after a dental procedure are the typical clinical manifestations of a masticator space abscess in adults4,5).

\section{Case Report:}

cially The case was a 65 -year-old female referred to us by dentist.

She had severe periodontitis of left lower third molar as diagnosed by the dentist with other chief complaint being swelling over left cheek and moderate trismus.patient was febrile and had toxic look making tooth extraction non feasible.

Lab findings were:-Hb-8.5 gm\%,TLC-13000/cmm,DLC-P85,L15;BSL-93mg\%;BUL/CREATININE23/0.9;Sr.BILI-0.8;SGOP/SGPT-32/38;Sr. PROTEIN/ALBUMIN-6.5/3.6.USG neck showed evidence of thick collection of pus in muscle plane in left cheek.We tried aspiration superfiin the region but failed. Patient was observed conservatively for 2 days but not responded to monocef and metronidazole and amox-clav. Then piperacillin tazobactum started but her condition worsened with swelling spreading to supra and infratemporal and periorbital region completely obscuring left eye opening.Then CT FACE $(\mathrm{P}+\mathrm{C})$ was done which showed left masticator space abscess.Surgical drainage of the deep abscess was done just below zygoma deep by gauge 18 no. needle with the help of CT report. Successful aspiration of pus was followed by an instantaneous improvement in the ability to open the mouth for a period of at least 24 hours. Pus culture and sensitivity was unyielding. After 2 days swelling over temporal region, incision and drainage of abscess in temporal region done. Patient was sent for tooth extraction.

\section{Discussion:}

Abscess formation in the orofacial region is relatively rare but it usually develops from an odontogenic location $(3,8)$. Odontogenic infections are commonly caused by pericoronitis, dental caries, periodontitis, or complications from dental procedures. The second and third molars are often the etiological tooth for these odontogenic infections ${ }^{(1)}$ A decayed tooth with an exposed pulp causes pulpitis, which, if untreated, develops into periodontitis.Pericoronitis or periodontitis can progress to alveolar ostitis or maxillare ostitis, which causes abscess formation in the orofacial region ${ }^{(7)}$.The masticator space is a distinct deep facial space, bounded by the superficial layer of the deep cervical fascia. It contains the ramus and posterior body of the mandible, and the 
four muscles of mastication, including the medial, lateral pterygoid muscle, temporal muscle and masseter muscle. This space is distinguished from other spaces by the superficial layer of deep cervical fascia. The space also contains several important nerves. These nerves, all branches of the mandibular division of the trigeminal nerve, include the masticator nerve, which innervates the muscles of mastication, as well as the buccal, lingual, and inferior alveolar nerves. Some clinical reports refer to the masticator space as temporal fossa and infratemporal fossa ${ }^{(2)}$. Masticator space is clinically important as a potential route of tumor progression and inflammation $\left({ }^{6}\right)$. It is commonly known that the contracture of medial and lateral pterygoid muscle in response to inflammation causes trismus and pain of TMJ.Muscle contracture or abscess formation in the masticator space tends to be recognized as a tumorous lesion by MRI views, however it is easy to discriminate between those diseases by CT imaging and the large amount of pus discharge observed during surgery. An abscess within the maxillo-mandibular bone must perforate jawbone itself before expanding to the surrounding soft tissues ${ }^{(5)}$. The least resistant, weakest and thinnest portion of the mandible is the lingual side of the molar region and the labial side of the anterior region. In the maxilla, the bone is the weakest on the labial or buccal side. An abscess, which perforates the buccal or labial plate of either the mandible or maxilla, will advance intraorally if it is disturbed by the bone attachments of the buccinator muscle, and extraorally if it perforates those muscular attachments. When a mandibular abscess perforates the lingual side of the molar region, it advances into the sublingual or submandibular spaces. A masticator abscess is usually caused by the advancement of a submandibular abscess. Normal flora in the oral cavity with low pathogenic potential can readily proliferate and cause abscess formation under immunosuppressive or ischemic or hypoxic conditions ${ }^{(4)}$. Bacterial products such as endotoxins, collagenase, fibrinolysin, elastase or hyaluronidase facilitate the inflammation ${ }^{(4)}$ Antibiotic therapy is important for preventing both local infective spread and bacteremia (1). The initial treatment for odontogenic disease is either an extraction or a root canal treatment of the affected tooth. If an advanced abscess is observed, the surgical drainage and debridement of necrotic tissue must be performed immediately. Before the surgical treatment, information concerning the anatomical spaces relating to the abscess formation is useful.Both CT and MRI provide reliable information regarding whether surgery is indicated or not, and which surgical approach is appropriate. These images are useful when the abscess is localized within the deep anatomical spaces such as the masticator space. An incision to the premature abscess disrupts the normal physiologic barriers and can cause further extension of the infection (5), whereas spontaneous abscess rupture through the skin damages the subcutaneous tissues and causes hypertrophic scars (5). In the cases of an abscess in the vestibular, buccal, pterygomandibular and canine space abscess, an intraoral incision at the appropriate time can prevent cutaneous scar formation. A submental or submandibular abscess requires an extraoral incision and drainage. The masticator space can be approached either intraorally or extraorally. The parapharyngeal space should therefore be approached extraorally to avoid any injury to the great vessels (6). In the present cases, the abscess was incised intraorally and a large amount of pus was drained from the abscess without any complications.Case had a TMJD due to a previous illness and thus developed a masticator abscess as a result of a tooth extraction, which confused the clinical findings and the diagnoses. The present cases could have been diagnosed by the high WBC count and CRP in blood analysis.In the cases of a deep masticator space abscess, the facial swelling or fervescence tends to be minor (1). However, a blood analysis is not always performed at the first visit in the cases of trismus with spontaneous pain of the TMJ. Consequently, when we treat TMJD, we must keep in mind the possibility of inapparent of the presence of some unseen inflammation, especially an abscess formation in the masticator space.

\section{Conclusion:}

Masticator space abscess is a rare entity to be kept in mind in every inflammatory neck swelling that is nonresponsive to treatment.It is secondary to caries of first and second molars mostly. CT face guides location and extent of abscess. Use of antibiotics alters clinical presentation and course therefore use of appropriate antibiotics is important but surgical intervention remains the cornerstone of treatment.

\section{References:}

[1] Bratton TA, Jackson DC, Nkungula-Howlett T, et al. 2002, Management of complex multi-space odontogenic infections. J Tenn Dent Assoc; 82(3): 39-47.

[2] Chong VF, Fan YF. 1996, Pictorial review: radiology of the masticator space. Clin Radiol; 51(7): 457-65.

[3] Chow AW, Roser SM, Brady FA. 1978, Orofacial odontogenic infections. Ann Intern Med; 88(3): 392-402

[4] Duran-Reynals F. 1942, TISSUE PERMEABILITY AND THE SPREADING FACTORS IN INFECTION: A Contribution to the Host:Parasite Problem. Bacteriol Rev; 6(4): 197-252.

[5] Endicott JN, Nelson RJ, Saraceno CA. 1982, Diagnosis and management decisions in infections of the deep fascial spaces of the head and neck utilizing computerized tomography. Laryngoscope; 92 (6 Pt 1): 630-3.

[6] Hardin CW, Harnsberger HR, Osborn AG, et al. 1985, Infection and tumor of the masticator space: CT evaluation. Radiology; 157(2): 413-417.

[7] Spilka CJ. 1966, Pathways of dental infections. J Oral Surg; 24(2): 111-24

[8] Welsh LW, Welsh JJ, Kelly JJ. 1991, Massive orofacial abscesses of dental origin. Ann Otol Rhinol Laryngol; 100(9 Pt 1): 768-773 
Fig1

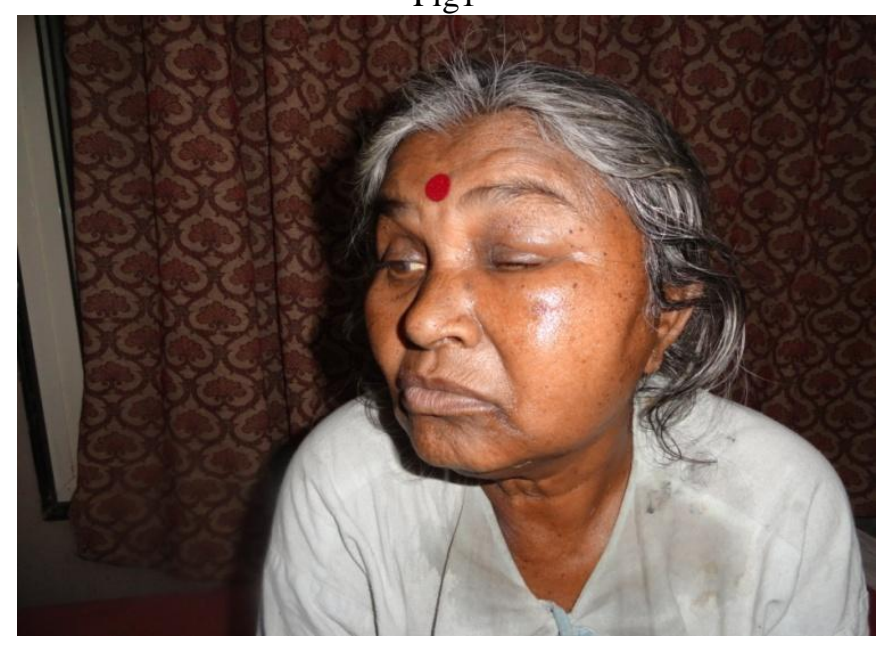

A case of masticator abscess showing supra temporal extension. (photograph on admission)

\section{Fig2}

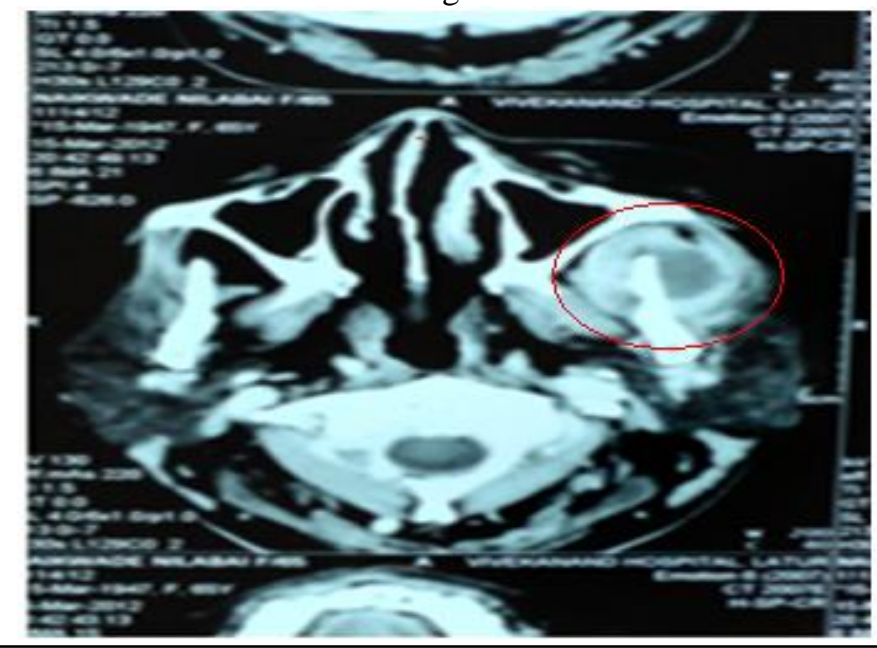

CT FACE-AXIAL section showing left masticator abscess pushing left temporalis muscle laterally and pterygoids mildly swollen.

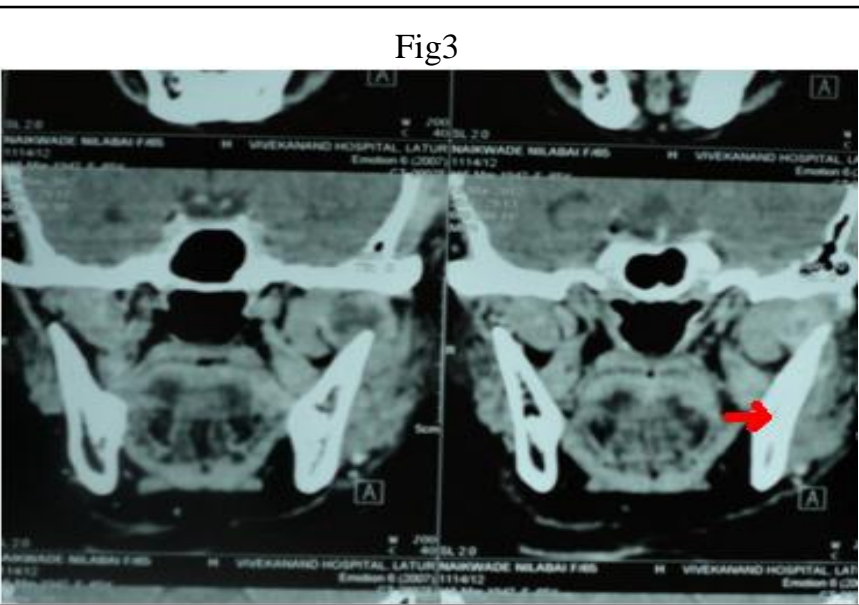

CT FACE CORONAL section showing spread of abscess to infratemporal region. Loss of medullary fat density of mandible (possibility of osteomyelitis) 


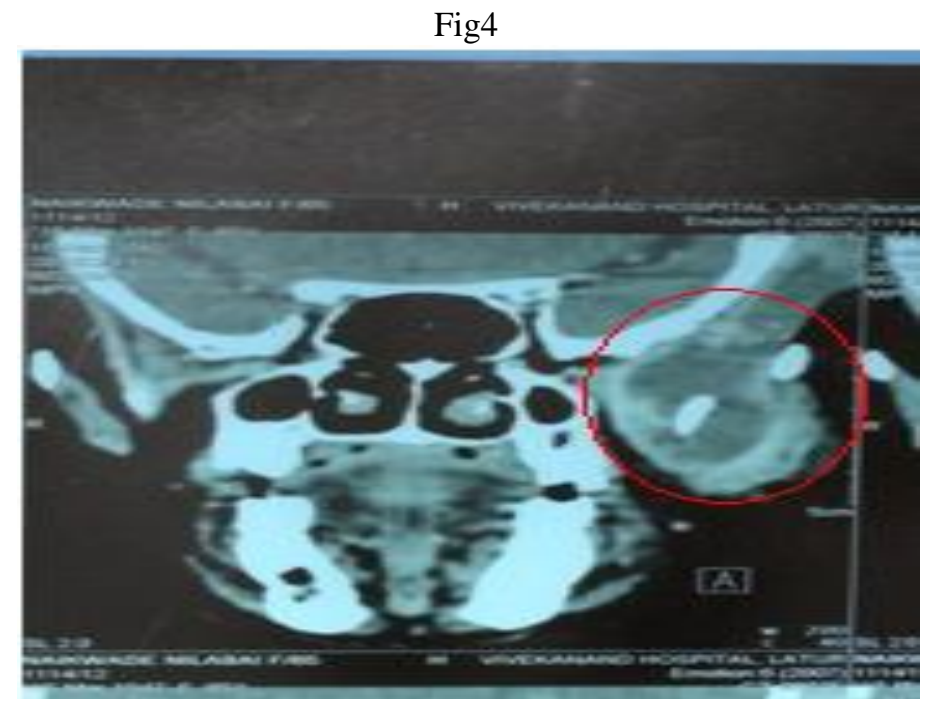

CT FACE CORONAL section showing supratemporal extension of masticator abscess.

Fig5

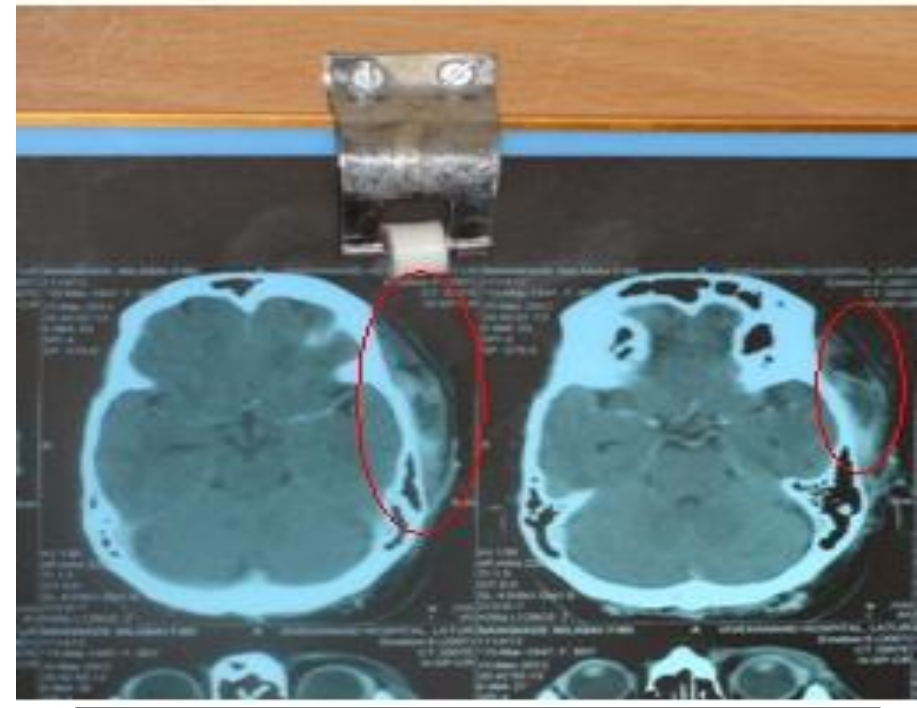

CT FACE AXIAL section showing small abscess cavity within temporalis muscle.

Fig6

Patient a day after surgical drainage.

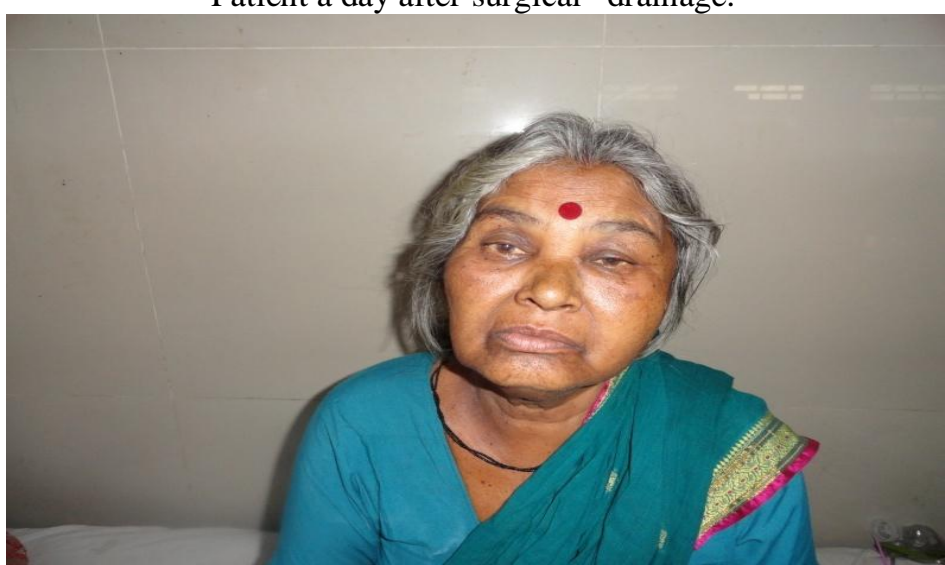

